\title{
Utilisation de la conjugaison complexe dans l'étude de la transcendance de valeurs de la fonction exponentielle usuelle
}

\author{
par GUY DIAZ
}

\begin{abstract}
RÉSUMÉ. Ce texte illustre l'usage que l'on peut faire de la conjugaison complexe en transcendance. Il montre aussi que la dérivation et le principe du maximum ne sont pas toujours des outils indispensables dans les preuves de transcendance. Ces deux constatations mises côte a côte permettront peut être de traiter quelques cas particuliers de la conjecture de Schanuel.
\end{abstract}

ABSTRACT. This paper illustrates the use we can make of complex conjugation in transcendence. It also proves that derivation and maximum modulus principle are not necessarily essential tools for transcendence proofs. These two facts put together might allow us to work on some particular cases of Schanuel's conjecture.

\section{Introduction}

Mon premier objectif est de souligner l'usage que l'on peut faire de la conjugaison complexe dans l'étude de la transcendance des valeurs de la fonction exponentielle usuelle ( en utilisant évidemment la propriété banale $e^{\bar{z}}=\overline{e^{z}}$ pour tout $\left.z \in \mathbb{C}\right)$. Je donnerai trois illustrations :

- ( $\$ 2$ ) trois résultats qui démontrent des cas particuliers de la conjecture des quatre exponentielles et de la conjecture forte des quatre exponentielles ;

- ( §3 ) une preuve nouvelle du théorème de Hermite - Lindemann restreint au cas où l'argument n'est pas dans $\mathbb{R} \cup i \mathbb{R}$;

- ( $§ 5$ ) deux conjectures qui sont des cas particuliers de la conjecture de Schanuel (et aussi des cas particuliers de la conjecture forte des quatre exponentielles) que je trouve particulièrement intéressantes.

Mon second objectif est de montrer que le recours dans les preuves de transcendance à des outils aussi classiques que la dérivation et le principe du maximum peut se révéler inutile, alors même qu'il constituait jusqu'ici

Manuscrit reçu le 1er avril 2003. 
un passage obligé. On verra ainsi ( $\S 4$ ) que le théorème de Hermite Lindemann restreint au cas où l'argument n'est pas dans $\mathbb{R} \cup i \mathbb{R}$ peut être démontré sans utilisation de la dérivation et du principe du maximum, mais avec recours à la conjugaison complexe. L'utilisation de la conjugaison complexe est illustrée ici à l'aide de la fonction exponentielle, mais n'est pas limitée à elle. Par exemple je l'avais utilisée dans [Di ] (voir le théorème 3 notamment) via la fonction modulaire $\mathrm{j}$.

La remarque que l'on peut souvent se passer du principe du maximum, du moins quand on s'intéresse à la fonction exponentielle, est une remarque faite à plusieurs reprises par Michel Waldschmidt et reprise dans son livre [Wa 3], §6.2.5 ("Avoiding the use of complex analysis"). Elle recoupe une remarque déjà ancienne (voir par exemple [Ge - Li ] , chap 12 ) énoncée ainsi par Michel Waldschmidt (voir [Wa 1]) :

"De nombreuses méthodes classiques de transcendance de nombres complexes utilisent des outils de la théorie des fonctions holomorphes. Quand on se restreint à l'étude de nombres réels, on peut effectuer ces démonstrations en n'employant dans la partie analytique que le théorème de Rolle".

Bien que les deux remarques se fassent écho elles sont distinctes : la première ne limite pas l'étude aux seuls nombres réels, ce que fait la seconde.

Quel est l'intérêt de proposer une nouvelle preuve du théorème de Hermite - Lindemann, avec qui plus est une restriction? Mon point de départ a été la conjoncture suivante (qui fait l'objet du §5) :

(?) Soit $u \in \mathbb{C} \backslash\{0\}$ avec $|u| \in \overline{\mathbb{Q}}$; alors $e^{u}$ est transcendant.

C'est bien sûr une conjecture qui généralise le théorème de Hermite Lindemann (on remplace l'hypothèse " $u \in \overline{\mathbb{Q}}$ " par l'hypothèse " $|u| \in \overline{\mathbb{Q}}$ "). Sa formulation m'incite à utiliser des fonctions comme $z \mapsto \bar{z}, z \mapsto|z|$ qui ne sont pas dérivables. Cela interdit alors l'usage de la dérivation et l'usage du principe du maximum. D'où la question naturelle : est-ce que l'on peut déjà démontrer le théorème de Hermite - Lindemann en se privant de ces outils classiques? La réponse donnée au $\S 4$, bien que partielle, est assez satisfaisante. Mais, pour l'instant, la conjecture ci-dessus résiste.

Je pense que l'on doit pouvoir donner d'autres exemples d'utilisations de la conjugaison complexe.

Pour simplifier la lecture j'ai surtout fait référence au livre de Michel Waldschmidt [Wa 3 ], dont je garde les notations. Ce texte doit beaucoup aux idées et travaux de Damien Roy et Michel Waldschmidt.

\section{Exemples de valeurs transcendantes de la fonction exponentielle usuelle}

Le premier exemple est la démonstration d'un cas particulier de la conjecture des quatre exponentielles, conjecture (notée $\mathrm{C} 4 \mathrm{E}$ ) qui s'énonce ainsi ( [Wa], p. 14) : 
C4E. Soient $x_{1}, x_{2}$ deux nombres complexes $\mathbb{Q}$ - linéairement indépendants et $y_{1}, y_{2}$ deux nombres complexes eux aussi $\mathbb{Q}$ - linéairement indépendants. Alors au moins un des quatre nombres exp $\left(x_{i} y_{j}\right), 1 \leq i, j \leq 2$, est transcendant.

Quitte à remplacer $\left(x_{1}, x_{2}, y_{1}, y_{2}\right) \operatorname{par}\left(x_{1} y_{1}, x_{2} y_{1}, 1, y_{2} / y_{1}\right)$ on peut se ramener au cas $y_{1}=1$ et les quatre exponentielles sont alors $e^{x_{1}}, e^{x_{2}}, e^{x_{1} y_{2}}$ et $e^{x_{2} y_{2}}$.

On va démontrer un cas particulier de cette conjecture, cas qui ne semblait pas connu jusqu'ici.

Théorème 1. Soient $x_{1}, x_{2}$ deux éléments de $\mathbb{R} \cup i \mathbb{R}, \mathbb{Q}$ - linéairement indépendants, et $y$ un nombre complexe non réel, de partie réelle irrationnelle. Alors au moins un des quatre nombres $e^{x_{1}}, e^{x_{2}}, e^{y x_{1}}, e^{y x_{2}}$ est transcendant.

L'idée est d'utiliser le théorème des six exponentielles (voir [Wa 3], p. 14 ) appliqué aux familles $\left(x_{1}, x_{2}\right)$ et $(1, y, \bar{y})$, les hypothèses faites sur $y$ permettant de dire que la famille $(1, y, \bar{y})$ est $\mathbb{Q}$ - libre (on voit ainsi que l'argument ne passe plus si $y$ est réel!).

Preuve du théorème 1.

Point 1 - Montrons que $1, y, \bar{y}$ sont $\mathbb{Q}$ - linéairement indépendants. Je suppose que $a, b, c$ sont dans $\mathbb{Q}$ et vérifient $a+b y+c \bar{y}=0$; je vais montrer que $a=b=c=0$. L'hypothèse donne :

$$
\left\{\begin{aligned}
a+(b+c) \operatorname{Re} y & =0 \\
(b-c) \operatorname{Im} y & =0 .
\end{aligned}\right.
$$

Puisque Im $y$ n'est pas nul, on en déduit $b=c$ et donc $: a+2 b(\operatorname{Re} y)=0$. Puisque $\operatorname{Re} y \notin \mathbb{Q}$ il en résulte que $a$ et $b$ sont nuls. C'est terminé.

Point 2 - On applique le théorème des six exponentielles aux familles $\left(x_{1}, x_{2}\right)$ et $(1, y, \bar{y})$. Cela permet de dire que :

$$
\operatorname{degtr}_{\mathbb{Q}} \mathbb{Q}\left(e^{x_{1}}, e^{x_{2}}, e^{y x_{1}}, e^{y x_{2}}, e^{\bar{y} x_{1}}, e^{\bar{y} x_{2}}\right) \geq 1 .
$$

Par ailleurs on a $\bar{x}_{1}=\varepsilon_{1} \cdot x_{1}, \bar{x}_{2}=\varepsilon_{2} . x_{2}$ avec $\varepsilon_{1}, \varepsilon_{2} \in\{1,-1\}$, par hypothèse.

Et en conséquence : $e^{\bar{y} x_{1}}=\overline{e^{\varepsilon_{1} y x_{1}}}, e^{\bar{y} x_{2}}=\overline{e^{\varepsilon_{2} y x_{2}}}$. Cela permet d'en déduire $\operatorname{degtr} \operatorname{tr}_{\mathbb{Q}}\left(e^{x_{1}}, e^{x_{2}}, e^{y x_{1}}, e^{y x_{2}}\right) \geq 1$ (sinon $e^{y x_{1}}, e^{y x_{2}}$ seraient algébriques, et donc aussi $e^{\bar{y} x_{1}}, e^{\bar{y} x_{2}}$ compte-tenu des relations de conjugaison, ce qui conduirait à une contradiction).

On peut noter que dans le théorème 1 il n'y a pas d'hypothèse d'algébricité pour les arguments $x_{1}, x_{2}, y$. Bien sûr, sous de telles hypothèses, les théorèmes classiques (comme les théorèmes de Hermite - Lindemann, de 
Gel'fond - Schneider) permettent d'obtenir des résultats plus précis. Par exemple si $x_{1}, x_{2}$ sont deux nombres complexes $\mathbb{Q}$ - linéairement indépendants avec $x_{1} / x_{2} \in \overline{\mathbb{Q}}$ alors pour tout $y \in \mathbb{C} \backslash\{0\}$ on a par le théorème de Gel'fond - Schneider ( voir $\S 3): \operatorname{degtr}_{\mathbb{Q}} \mathbb{Q}\left(e^{y x_{1}}, e^{y x_{2}}\right) \geq 1$.

On peut proposer des variantes du théorème 1 ; par exemple on peut remplacer le couple $\left(x_{1}, x_{2}\right)$ par un couple $(x, \bar{x})$ où $x \in \mathbb{C} \backslash(\mathbb{R} \cup i \mathbb{R})$ sans changer l'hypothèse sur $y$ et on obtient : $\operatorname{degtr} \mathbb{Q} \mathbb{Q}\left(e^{x}, e^{x y}, e^{x \bar{y}}\right) \geq 1$.

Le second exemple est la démonstration d'un cas particulier de la conjecture forte des quatre exponentielles (conjecture 11.17 de [Wa 3]) à partir du théorème fort des six exponentielles ([Wa 3], p. 399). Rappelons les notations de [Wa 3 ] :

* $\mathcal{L}$ est le $\mathbb{Q}$ - espace vectoriel des logarithmes de nombres algébriques, c'est à dire

$$
\mathcal{L}:=\{\lambda \in \mathbb{C} ; \exp (\lambda) \in \overline{\mathbb{Q}}\}
$$

* $\widetilde{\mathcal{L}}$ est le $\overline{\mathbb{Q}}$ - espace vectoriel engendré, dans $\mathbb{C}$, par 1 et $\mathcal{L}$.

Théorème 2. Soit y un nombre complexe pour lequel la famille $(1, y, \bar{y})$ est $\overline{\mathbb{Q}}$ - libre . Soient $x_{1}$ et $x_{2}$ deux nombres complexes $\overline{\mathbb{Q}}$ linéairement indépendants; on suppose que

$$
\left\{\begin{array}{l}
\text { soit } x_{1} / \bar{x}_{1} \text { et } x_{2} / \bar{x}_{2} \text { sont algébriques } \\
\text { soit } x_{1} / \bar{x}_{2} \text { est algébrique. }
\end{array}\right.
$$

Alors au moins un des quatre nombres $x_{1}, x_{2}, x_{1} y, x_{2} y$ n'est pas dans $\widetilde{\mathcal{L}}$.

Preuve du théorème 2.

On applique le théorème fort des six exponentielles aux familles $\left(x_{1}, x_{2}\right)$ et $(1, y, \bar{y}) ;$ il permet de dire que :

$$
\left\{x_{1}, x_{2}, x_{1} y, x_{2} y, x_{1} \bar{y}, x_{2} \bar{y}\right\} \not \subset \tilde{\mathcal{L}} \text {. }
$$

Je suppose $\left\{x_{1}, x_{2}, x_{1} y, x_{2} y\right\} \subset \widetilde{\mathcal{L}}$ et cela conduit à une contradiction car alors $x_{1} \bar{y}$ et $x_{2} \bar{y}$ sont aussi éléments de $\widetilde{\mathcal{L}}$. En effet :

${ }^{*}$ si $\beta_{1}:=x_{1} / \bar{x}_{1}$ et $\beta_{2}:=x_{2} / \bar{x}_{2}$ sont algébriques on a $x_{1} y=\beta_{1} \bar{x}_{1} y, x_{2} y=$ $\beta_{2} \bar{x}_{2} y$; comme $x_{1} y \in \widetilde{\mathcal{L}}, \beta_{1} \in \overline{\mathbb{Q}} \backslash\{0\}$, on en tire $\bar{x}_{1} y \in \widetilde{\mathcal{L}}$ donc $x_{1} \bar{y} \in \widetilde{\mathcal{L}}$ ( car $\mathcal{L}$ et $\widetilde{\mathcal{L}}$ sont stables par conjugaison complexe). Même chose pour $x_{2} \bar{y}$.

* Si $\alpha:=x_{1} / \bar{x}_{2}$ est algébrique on a $x_{1}=\alpha \bar{x}_{2}, \bar{x}_{1}=\bar{\alpha} x_{2}$; donc $x_{1} y=$ $\alpha \overline{x_{2}} y, x_{2} y=(1 / \bar{\alpha}) \bar{x}_{1} y$ et on termine comme au premier point.

D'autres variantes sont possibles. En voici une qui fournit toute une famille de nombres transcendants; pour des nombres ayant la même forme le résultat donné dans [Wa 3 ], p. 385 , dernier paragraphe, ne fournit que la transcendance d'un nombre parmi deux. 
Théorème 3. Soient $\lambda_{0} \in \widetilde{\mathcal{L}} \backslash \overline{\mathbb{Q}}$ et $\lambda_{1} \in \mathcal{L} \backslash\{0\}$ avec $\lambda_{1} \notin \mathbb{R} \cup i \mathbb{R}$.

- 1) Alors au moins un des nombres $\lambda_{0} \lambda_{1}$ et $\bar{\lambda}_{0} \lambda_{1}$ n'est pas dans $\widetilde{\mathcal{L}}$.

- 2) En particulier si $\lambda_{0} \in \mathbb{R} \cup i \mathbb{R}$, le nombre $\lambda_{0} \lambda_{1}$ n'est pas dans $\widetilde{\mathcal{L}}$ (en conséquence $\lambda_{0} \lambda_{1}$ est transcendant et pour tout $\beta \in \overline{\mathbb{Q}} \backslash\{0\}$, exp $\left(\beta \lambda_{0} \lambda_{1}\right)$ est transcendant); et le nombre $\lambda_{1} / \lambda_{0}$ n'est pas dans $\mathcal{L}$.

On conjecture que sous la seule hypothèse " $\lambda_{0}, \lambda_{1} \in \mathcal{L} \backslash\{0\}$ " les nombres $\lambda_{0} \lambda_{1}$ et $e^{\lambda_{0} \lambda_{1}}$ sont transcendants (ce qui en particulier donnerait la transcendance de $\left.\exp \left(\pi^{2}\right)\right)$. Le théorème 3 donne une famille d'exemples où c'est bien ce qui se passe, mais il ne dit rien de $\exp \left(\pi^{2}\right)$.

Démonstration. Preuve du théorème 3

La famille $\left(1, \lambda_{0}\right)$ est $\overline{\mathbb{Q}}$ - libre puisque $\lambda_{0}$ est transcendant. L'hypothèse $\lambda_{1} \notin \mathbb{R} \cup i \mathbb{R}$ dit que la famille $\left(\lambda_{1}, \overline{\lambda_{1}}\right)$ est $\mathbb{Q}$ - libre ; comme $\lambda_{1}$ et $\bar{\lambda}_{1}$ sont des logarithmes de nombres algébriques, le théorème de Baker ([Wa 3], theorem 1.6, p. 3) permet de dire que la famille $\left(1, \lambda_{1}, \overline{\lambda_{1}}\right)$ est $\overline{\mathbb{Q}}$ - libre.

-1) Le théorème fort des six exponentielles appliqué aux familles $\left(1, \lambda_{0}\right)$ et $\left(1, \lambda_{1}, \overline{\lambda_{1}}\right)$ permet de dire que l'on a :

$$
\left\{1, \lambda_{0}, \lambda_{1}, \overline{\lambda_{1}}, \lambda_{0} \lambda_{1}, \lambda_{0} \overline{\lambda_{1}}\right\} \not \subset \widetilde{\mathcal{L}} \text {. }
$$

Comme $1, \lambda_{0}, \lambda_{1}, \overline{\lambda_{1}}$ sont éléments de $\widetilde{\mathcal{L}}$, on peut conclure que $\lambda_{0} \lambda_{1}$ ou $\lambda_{0} \bar{\lambda}_{1}$ n'est pas dans $\widetilde{\mathcal{L}}$. Et $\widetilde{\mathcal{L}}$ est stable par conjugaison complexe ce qui permet de remplacer $\lambda_{0} \overline{\lambda_{1}}$ par $\overline{\lambda_{0}} \lambda_{1}$ dans la conclusion précédente.

- 2) On suppose en plus $\lambda_{0} \in \mathbb{R} \cup i \mathbb{R}$. On a donc $\overline{\lambda_{0}}= \pm \lambda_{0}, \overline{\lambda_{0}} \lambda_{1}= \pm \lambda_{0} \lambda_{1}$. Et donc le point 1 permet de conclure $\lambda_{0} \lambda_{1} \notin \widetilde{\mathcal{L}}$. Pour $\lambda_{1} / \lambda_{0}$, on suppose que $\lambda:=\lambda_{1} / \lambda_{0}$ est dans $\mathcal{L}$; on a donc $\lambda_{1}=\lambda \lambda_{0}$ et on peut affirmer que $\lambda \notin \mathbb{R} \cup i \mathbb{R}$. Mais on vient de voir qu'alors $\lambda \lambda_{0}$ ne peut pas être dans $\mathcal{L} ;$ c'est contradictoire .

\section{A propos du théorème de Hermite - Lindemann}

Je vais démontrer le théorème suivant, que j'appelerai théorème de Hermite - Lindemann restreint (HLR), à partir du théorème de Gel'fond Schneider.

Théorème 4 (Théorème HLR). Soit $\alpha$ un nombre algébrique n'appartenant pas à $\mathbb{R} \cup i \mathbb{R}$. Alors pour tout nombre réel $v$ non nul, $e^{v \alpha}$ est transcendant. En particulier $e^{\alpha}$ est transcendant.

Le vrai théorème de Hermite-Lindemann concerne le cas général $\alpha \in$ $\overline{\mathbb{Q}} \backslash\{0\}$ et affirme que $e^{\alpha}$ est transcendant. L'hypothèse $\alpha \notin \mathbb{R} \cup i \mathbb{R}$ permet de dire que la famille $(\alpha, \bar{\alpha})$ est $\mathbb{Q}$ - libre et donc d'introduire le conjugué $\bar{\alpha}$ : elle sert à ça et uniquement à ça. 
La première assertion du théorème 4 n'est plus vraie si $\alpha \in(\mathbb{R} \cup i \mathbb{R}) \cap \overline{\mathbb{Q}}$ (pour $\alpha \in \overline{\mathbb{Q}} \cap \mathbb{R}, \alpha \neq 0$, prendre $v:=(\log 2) / \alpha$; pour $\alpha \in \overline{\mathbb{Q}} \cap i \mathbb{R}, \alpha \neq 0$, prendre $v:=2 i \pi / \alpha$ ). Ce qui montre au passage que les nombres algébriques n'appartenant pas à $\mathbb{R} \cup i \mathbb{R}$ n'ont pas, du point de vue de la transcendance, les mêmes propriétés que ceux de $\mathbb{R} \cup i \mathbb{R}$. C'est surprenant.

On peut énoncer le théorème 4 ainsi : l'image, par exp, de la droite $\mathbb{R} \alpha$ privée de 0 est une courbe ( réelle ) dans $\mathbb{C}$ dont tous les points sont transcendants.

En contraposant on peut dire ça ainsi : si $\lambda$ est un logarithme de nombre algébrique ( ie $\exp (\lambda) \in \overline{\mathbb{Q}})$ n'appartenant pas à $\mathbb{R} \cup i \mathbb{R}$, alors la droite $\mathbb{R} \lambda$ privée de l'origine est constituée uniquement de points transcendants (soit $t \in \mathbb{R} \backslash\{0\}$ tel que $\alpha:=t \lambda$ soit algébrique; on sait par le théorème 4 que $\exp \left(\frac{1}{t} \alpha\right)$ est transcendant, ce qui est contradictoire puisque $\left.\frac{1}{t} \alpha=\lambda\right)$.

Le théorème 4 est un corollaire du théorème de Gel'fond - Schneider, que je vais rappeler (voir [Wa 2 ] p. 42 , p. 188).

Théorème (Gel'fond - Schneider). Soit $u_{1}, u_{2}, v$ des nombres complexes non nuls. Si $u_{1}, u_{2}$ sont $\mathbb{Q}$-linéairement indépendants alors un des trois nombres $u_{2} / u_{1}, e^{v u_{1}}, e^{v u_{2}}$ est transcendant.

Preuve du théorème 4.

Les hypothèses et notations sont celles du théorème 4 . Puisque $\alpha \notin \mathbb{R} \cup i \mathbb{R}$ la famille $(\alpha, \bar{\alpha})$ est $\mathbb{Q}$ - libre; le théorème de Gel'fond - Schneider appliqué à $\left(u_{1}, u_{2}\right):=(\alpha, \bar{\alpha})$ dit alors que

$$
\operatorname{degtr}_{\mathbb{Q}} \mathbb{Q}\left(e^{v \alpha}, e^{v \bar{\alpha}}\right) \geq 1 .
$$

Puisque $v$ est réel on sait que $e^{v \bar{\alpha}}=e^{\overline{v \alpha}}=\overline{e^{v \alpha}}$; donc si $e^{v \alpha}$ était algébrique $e^{v \bar{\alpha}}$ le serait aussi et on aurait une contradiction. C'est donc que $e^{v \alpha}$ est transcendant.

Commentaire sur cette preuve. Je ne veux pas réécrire l'histoire du théorème de Gel'fond - Schneider, mais il faut avoir à l'esprit le fait que l'on en connait essentiellement deux preuves : celle de Gel'fond qui utilise l'équation différentielle satisfaite par la fonction exponentielle et donc qui utilise la dérivation; et celle de Schneider qui n'utilise pas la dérivation (voir par exemple [Wa 2] , chapitres 2 et 3). Du coup cela veut dire que l'on peut démontrer le théorème 4 sans utiliser la dérivation! Je trouve cela assez étonnant dans la mesure où jusqu'ici le théorème de Hermite - Lindemann était très étroitement associé aux propriétés de dérivabilité de exp. Je reviendrai la dessus au paragraphe suivant.

Cela conduit à poser la question suivante :

(? ) Peut-on démontrer le théorème de Hermite - Lindemann (le vrai) sans utiliser la dérivation? 
Cela me semble impossible; et pourtant le théorème suivant dit que l'on n'est pas très loin de pouvoir conclure.

Théorème 5. Le théorème de Gel'fond - Schneider permet de dire que l'ensemble $\left\{\alpha \in \overline{\mathbb{Q}} \backslash\{0\} ; e^{\alpha} \in \overline{\mathbb{Q}}\right\}$ est soit vide, soit de la forme $\mathbb{Q}^{*} \alpha_{0}$ (avec $\left.\alpha_{0} \in \mathbb{R} \cup i \mathbb{R}\right)$.

Dit autrement, le théorème de Hermite - Lindemann se déduit du théorème de Gel'fond - Schneider " à au plus une exception près "; il n'y a pas beaucoup de cas qui échappent, mais il y en a encore!

\section{Preuve du théorème 5.}

Je note $\mathcal{A}:=\left\{\alpha \in \overline{\mathbb{Q}} \backslash\{0\} ; e^{\alpha} \in \overline{\mathbb{Q}}\right\}$ et je suppose $\mathcal{A}$ non vide. Soit $\alpha_{0}$ un élément de $\mathcal{A}$ ( le théorème 4 dit alors que $\alpha_{0} \in \mathbb{R} \cup i \mathbb{R}$ ). Montrons que $\mathcal{A}=\mathbb{Q}^{*} \alpha_{0}$. L'inclusion $\mathbb{Q}^{*} \alpha_{0} \subset \mathcal{A}$ est évidente. Pour l'autre inclusion, je pars de $\beta \in \mathcal{A} ;$ je suppose $\beta \notin \mathbb{Q} \alpha_{0}$ et je cherche une contradiction. La famille $\left(\alpha_{0}, \beta\right)$ est $\mathbb{Q}$ - libre et alors le théorème de Gel'fond - Schneider dit que l'un des trois nombres $\alpha_{0} / \beta, e^{\alpha_{0}}, e^{\beta}$ est transcendant, ce qui est faux.

\section{Une preuve du théorème HLR n'utilisant ni dérivation, ni principe du maximum}

Comme annoncé, je vais donner une preuve détaillée du théorème 4 (HLR) n'utilisant ni dérivation, ni principe du maximum. J'aurais aussi bien pu donner une preuve du théorème de Gel'fond - Schneider mais je préfêre rester le plus proche possible de la conjecture évoquée au $(\S 1)$.

Ma preuve va simplement consister à expliciter, dans le cas qui m'intéresse, une remarque de $\mathrm{M}$. Waldschmidt ([Wa 3 ] , §6.2.5, p. 180) :

“... The proofs we give in these lectures do not require any complex analysis ... The only point where analysis played any role so far was in the use of Schwarz'lemma 2.4, in the proof of lemma 6.3. But we use it only for exponential polynomials in one variable, and in this case the estimate is quite easy."

Plutôt que de dire que je n'utilise pas l'analyse complexe, j'ai choisi de dire que je n'utilise pas le principe du maximum ( et à fortiori pas le "lemme de Schwarz"). A première lecture la portée de la remarque de M. Waldschmidt n'est pas très claire : les preuves en question sont-elles celles du chapitre 6 (qui porte sur le ihéorème de Baker, cas homogène et cas non homogène) ou celles de tout le livre? En fait cette remarque s'applique au théorème de Baker (cas homogène et cas non homogène) mais aussi au théorème des six exponentielles, c'est à dire finalement à tous les théorèmes classiques sur la transcendance de valeurs de la fonction exponentielle. 
La preuve repose sur la méthode des déterminants d'interpolation de $\mathrm{M}$. Laurent (la méthode des alternants dans le langage de $\mathrm{M}$. Waldschmidt, puisqu'il n'y a pas de dérivation). Cette approche, introduite par M Laurent [La], est systématiquement utilisée par M. Waldschmidt dans [Wa 3] (où elle est présentée en détails au paragraphe 2.1).

\subsection{Résultats préliminaires.}

Ce paragraphe rassemble des outils standards des preuves de transcendance : un lemme de zéros (lemme 1), un lemme qui jouera le rôle du "lemme de Schwarz" (lemme 2) et l'inégalité de Liouville (lemme 4). Les notations utilisées sont celles de [Wa 3] et je ne les redéfinis pas.

Le lemme 1 est un lemme de zéros dû à Y.V. Nesterenko, dans la version donnée par M. Waldschmidt ( voir [Wa 3], proposition 2.12, p.52). La preuve en est purement algébrique.

Lemme 1. Soient $K$ un corps de caractéristique nulle, $D_{0}$ et $D_{1}$ des entiers strictement positifs, $\left(x_{1}, y_{1}\right), \ldots,\left(x_{M_{1}}, y_{M_{1}}\right),\left(\xi_{1}, \eta_{1}\right), \ldots,\left(\xi_{M_{2}}, \eta_{M_{2}}\right)$ des éléments de $K \times(K \backslash\{0\})$ où $y_{1}, \ldots, y_{M_{1}}$ sont deux à deux distincts et $\xi_{1}, \ldots, \xi_{M_{2}}$ sont deux à deux distincts. On suppose $M_{1}>D_{1}$ et $M_{2}>$ $D_{0}\left(1+D_{1}\right)$. Alors il n'existe pas de polynôme non nul $P \in K\left[X_{0}, X_{1}\right]$ vérifiant :

$$
\left\{\begin{array}{l}
. \operatorname{deg}_{X_{0}}(P) \leq D_{0} \text { et } \operatorname{deg}_{X_{1}}(P) \leq D_{1} \\
P\left(x_{\nu}+\xi_{\mu}, y_{\nu} \eta_{\mu}\right)=0 \text { pour } 1 \leq \nu \leq M_{1}, 1 \leq \mu \leq M_{2}
\end{array}\right.
$$

Le lemme 2 est un lemme (élémentaire dans sa preuve) qui permet de retrouver pour les polynômes exponentiels l'estimation que l'on obtiendrait par le classique lemme de Schwarz, mais sans avoir à invoquer le principe du maximum. C'est le lemme 6.8 de [Wa 3], p. 180.

Lemme 2. Soient $a_{i j}$ (pour $0 \leq i \leq s, 1 \leq j \leq t$ ) et $w_{j}$ (pour $1 \leq j \leq t$ ) des nombres complexes. Je suppose que le polynôme exponentiel

$$
F(z):=\sum_{i=0}^{s} \sum_{j=1}^{t} a_{i j} \cdot z^{i} \cdot e^{w_{j} z}, z \in \mathbb{C}
$$

a un zéro à l'origine de multiplicité supérieure ou égale à $T$. Alors pour $z_{0} \in \mathbb{C}$ et $R \geq\left|z_{0}\right|>0$ on $a:$

$$
\left|F\left(z_{0}\right)\right| \leq\left(\frac{R}{\left|z_{0}\right|}\right)^{-T} \sum_{i=0}^{s} \sum_{j=1}^{t}\left|a_{i j}\right| \cdot R^{i} \cdot e^{\left|w_{j}\right| R}
$$

Preuve du lemme 2.

- En reportant $e^{w_{j} z}=\sum_{k=0}^{+\infty} \frac{1}{k !}\left(w_{j} z\right)^{k}$ dans $F(z)$ on peut écrire : 


$$
F(z)=\sum_{n=0}^{+\infty} \alpha_{n} z^{n} \quad \text { avec } \quad \alpha_{n}=\sum_{i=0}^{\min (s, n)} \sum_{j=1}^{t} a_{i j} \frac{w_{j}^{n-i}}{(n-i) !}
$$

(justification : $F(z)=\sum_{i=0}^{s} \sum_{j=1}^{t} a_{i j} \cdot z^{i}\left(\sum_{k=0}^{+\infty} \frac{w_{j}^{k}}{k !} z^{k}\right)=\sum_{k=0}^{+\infty} \sum_{i=0}^{s} \sum_{j=1}^{t} a_{i j} \frac{w_{j}^{k}}{k !} z^{i+k}$

donc le coefficient $\alpha_{n}$ de $z^{n}$ s'obtient en considérant tous les triplets $(i, j, k)$ avec $i+k=n$, c'est à dire tous les triplets $(i, j, n-i)$; comme on doit de plus avoir $0 \leq i \leq s$ et $0 \leq n-i$, cela limite l'indice $\mathrm{i}$ à $0 \leq i \leq \min (n, s)$ et cela donne l'expression (1) de $\alpha_{n}$ ).

- Ce même calcul avec $\left|a_{i j}\right|$ à la place de $a_{i j}$ et $\left|w_{j}\right|$ à la place de $w_{j}$ donne :

$$
\sum_{i=0}^{s} \sum_{j=1}^{t}\left|a_{i j}\right| \cdot z^{i} \cdot e^{\left|w_{j}\right| z}=\sum_{n=0}^{+\infty}\left(\sum_{i=0}^{\min (n, s)} \sum_{j=1}^{t}\left|a_{i j}\right| \frac{\left|w_{j}\right|^{n-i}}{(n-i) !}\right) \cdot z^{n}
$$

- Par hypothèse $F$ a un zéro à l'origine de multiplicité $\geq T$ ce qui se traduit par $\alpha_{n}=0$ pour $0 \leq n \leq T-1$, c'est à dire encore $F(z)=$ $\sum_{n=T}^{+\infty} \alpha_{n} . z^{n}$. Et pour $n \geq T$ on a $\left(\frac{R}{\left|z_{0}\right|}\right)^{T} \leq\left(\frac{R}{\left|z_{0}\right|}\right)^{n}$ puisqu'on a supposé

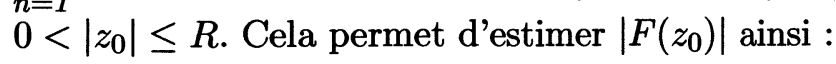

$$
\begin{aligned}
\left|F\left(z_{0}\right)\right| & \leq \sum_{n=T}^{+\infty}\left|\alpha_{n}\right| \cdot\left|z_{0}\right|^{n} \\
& \leq\left(\frac{\left|z_{0}\right|}{R}\right)^{T} \cdot \sum_{n=T}^{+\infty}\left|\alpha_{n}\right| \cdot\left|z_{0}\right|^{n-T} \cdot R^{T}
\end{aligned}
$$

Comme on a $\left|z_{0}\right| \leq R$ on peut majorer $\left|z_{0}\right|^{n-T} R^{T}$ par $R^{n}$ et il reste :

$$
\left|F\left(z_{0}\right)\right| \leq\left(\frac{\left|z_{0}\right|}{R}\right)^{T} \cdot \sum_{n=T}^{+\infty}\left|\alpha_{n}\right| \cdot R^{n}
$$

En utilisant (1) on obtient :

$$
\begin{aligned}
\sum_{n=T}^{+\infty}\left|\alpha_{n}\right| \cdot R^{n} & \leq \sum_{n=T}^{+\infty} \sum_{i=0}^{\min (s, t)} \sum_{j=1}^{t}\left|a_{i j}\right| \frac{\left|w_{j}\right|^{n-i}}{(n-i) !} R^{n} \\
& \leq \sum_{n=0}^{+\infty} \sum_{i=0}^{\min (s, t)} \sum_{j=1}^{t}\left|a_{i j}\right| \frac{\left|w_{j}\right|^{n-i}}{(n-i) !} R^{n}
\end{aligned}
$$


Ce qui grâce à (2) s'écrit :

$$
\sum_{n=T}^{+\infty}\left|\alpha_{n}\right| \cdot R^{n} \leq \sum_{i=0}^{s} \sum_{j=1}^{t}\left|a_{i j}\right| R^{i} e^{\left|w_{j}\right| R}
$$

En reportant dans (3) cela donne :

$$
\left|F\left(z_{0}\right)\right| \leq\left(\frac{\left|z_{0}\right|}{R}\right)^{T} \sum_{i=0}^{s} \sum_{j=1}^{t}\left|a_{i j}\right| \cdot R^{i} . e^{\left|w_{j}\right| R}
$$

On voit au demeurant que la démarche utilisée a un caractère très général et doit pouvoir s'adapter à bien d'autres fonctions que les polynômes exponentiels. Il est donc fort probable que ce qui est dit dans ce $\S 4$ pour la fonction exponentielle peut se transposer à d'autres fonctions.

Lemme 3. Soient $\varphi_{1}, \ldots, \varphi_{L}$ des fonctions analytiques sur $\mathbb{C}$ et $z_{1}, \ldots, z_{L}$ des nombres complexes. La fonction analytique

$$
\psi(z):=\operatorname{det}\left(\varphi_{\lambda}\left(z . z_{\mu}\right)\right)_{1 \leq \lambda, \mu \leq L}
$$

a un zéro à l'origine de multiplicité supérieure ou égale à $L(L-1) / 2$.

La preuve est le (a) de la preuve du lemme 2.5, p. 37, de [Wa 3]. Elle n'utilise que la multi-linéarité du déterminant et le développement à l'origine des $\varphi_{\lambda}$.

Le dernier lemme est une inégalité de Liouville (voir [Wa 3], p. 83). Je rappelle que pour un polynôme $Q$ on note $L(Q)$ sa longueur, c'est-à-dire la somme des valeurs absolues de ses coefficients; et que pour un nombre algébrique $\alpha, h(\alpha)$ désigne la hauteur logarithmique absolue de $\alpha$.

Lemme 4. Soient $Q \in \mathbb{Z}\left[X_{1}, \ldots, X_{n}\right]$ et $\alpha_{1}, \ldots, \alpha_{n}$ des éléments d'un corps de nombres de degré $d$. Si $Q\left(\alpha_{1}, \ldots, \alpha_{n}\right)$ est non nul on a :

$$
\log \left|Q\left(\alpha_{1}, \ldots, \alpha_{n}\right)\right| \geq-d \cdot \log L(Q)-d \cdot \sum_{j=1}^{n} h\left(\alpha_{j}\right) \cdot \operatorname{deg}_{X_{j}}(Q)
$$

\subsection{Preuve du théorème 4 .}

On se place dans les hypothèses du théorème 4 : on se donne $\alpha \in \overline{\mathbb{Q}}, \alpha \notin$ $\mathbb{R} \cup i \mathbb{R}$, et $v \in \mathbb{R} \backslash\{0\}$. On suppose $e^{v \alpha}$ algébrique et on cherche une contradiction; notons que $e^{v \bar{\alpha}}$ est lui aussi algébrique.

- Notations.

Pour $t=\left(t_{0}, t_{1}\right) \in \mathbb{N}^{2}, \Phi_{t}$ est la fonction définie par $\Phi_{t}(z):=z^{t_{0}} \cdot e^{t_{1} v z}$. Pour $s=\left(s_{1}, s_{2}\right) \in \mathbb{N}^{2}$, je note $z_{s}:=s_{1} \alpha+s_{2} \bar{\alpha}$. 
On voit ainsi que les fonctions $\Phi_{t}$ prennent des valeurs algébriques en les points $z_{s}$ (puisque $\Phi_{t}\left(z_{s}\right)=\left(s_{1} \alpha+s_{2} \bar{\alpha}\right)^{t_{0}} \cdot\left(e^{v \alpha}\right)^{t_{1} s_{1}} \cdot\left(e^{v \bar{\alpha}}\right)^{t_{1} s_{2}}$ ) .

Les paramètres seront les entiers strictement positifs $T_{0}, T_{1}, S$. On leur associe les ensembles $\mathcal{T}$ et $\mathcal{S}$ suivants :

$$
\begin{aligned}
& \mathcal{T}:=\left\{\left(t_{0}, t_{1}\right) \in \mathbb{N}^{2} ; 0 \leq t_{0}<T_{0}, 0 \leq t_{1}<T_{1}\right\}, \\
& \mathcal{S}:=\left\{\left(s_{1}, s_{2}\right) \in \mathbb{N}^{2} ; 1 \leq s_{1}, s_{2} \leq 2 S\right\} .
\end{aligned}
$$

$\mathcal{M}$ sera la matrice à $T_{0} T_{1}$ lignes et $4 S^{2}$ colonnes données par :

$$
\mathcal{M}=\left(\Phi_{t}\left(z_{s}\right)\right)_{t \in \mathcal{T}, s \in \mathcal{S}}
$$

( $t$ est l'indice de ligne, $s$ est l'indice de colonne; et on suppose avoir ordonné les ensembles $\mathcal{T}$ et $\mathcal{S}$ ).

\section{- Premier point - Une condition suffisante pour que $\mathcal{M}$ soit de rang maximum.}

- (a) Je suppose que $\mathcal{M}$ n'est pas de rang maximum. Alors il existe une famille de nombres complexes non tous nuls $\left\{p_{t} ; t \in \mathcal{T}\right\}$ telle que la combinaison linéaire des lignes de $\mathcal{M}$ associée à cette famille de coefficients soit nulle :

$$
\sum_{t \in \mathcal{T}} p_{t} . \Phi_{t}\left(z_{s}\right)=0 \text { pour tout } s \in \mathcal{S}
$$

En introduisant le polynôme non nul $P\left(X_{0}, X_{1}\right):=\sum_{t \in \mathcal{T}} p_{t} \cdot X_{0}^{t_{0}} \cdot X_{1}^{t_{1}}$ cela s'écrit :

$$
P\left(s_{1} \alpha+s_{2} \bar{\alpha},\left(e^{v \alpha}\right)^{s_{1}} \cdot\left(e^{v \bar{\alpha}}\right)^{s_{2}}\right)=0 \text { pour tout } s \in \mathcal{S} .
$$

On se retrouve ainsi dans la situation décrite par le lemme 1. Et celui-ci va donner une condition suffisante pour que (4) soit impossible.

. (b) On applique le lemme 1 avec $K:=\mathbb{C}, D_{0}:=T_{0}-1, D_{1}:=T_{1}-$ $1, M_{1}:=S, M_{2}:=S^{2}$. On prend pour les $\left(\xi_{\mu}, \eta_{\mu}\right)$ les $M_{2}=S^{2}$ points de l'ensemble $\left\{\left(s_{1} \alpha+s_{2} \bar{\alpha},\left(e^{v \alpha}\right)^{s_{1}} \cdot\left(e^{v \bar{\alpha}}\right)^{s_{2}}\right) ; 1 \leq s_{1}, s_{2}, \leq S\right\}$; les $\xi_{\mu}$ (ie les $\left.s_{1} \alpha+s_{2} \bar{\alpha}\right)$ sont deux à deux distincts puisque $(\alpha, \bar{\alpha})$ est $\mathbb{Q}$ - libre .

On prend pour les $\left(x_{\nu}, y_{\nu}\right)$ les $M_{1}=S$ points de l'ensemble $\left\{\left(s_{1} \alpha,\left(e^{v \alpha}\right)^{s_{1}}\right)\right.$; $\left.1 \leq s_{1} \leq S\right\}$. Il faut justifier le fait que les $y_{\nu}$ sont deux à deux distincts, c'est à dire que l'application $s \in \mathbb{N} \mapsto\left(e^{v \alpha}\right)^{s}$ est injective; c'est immédiat compte tenu du fait que $\alpha \notin i \mathbb{R}$ et $v \neq 0$. Ainsi (4) permet de dire que l'on $\mathrm{a}:$

$$
P\left(x_{\nu}+\xi_{\mu}, y_{\nu} \cdot \eta_{\mu}\right)=0 \text { pour } 1 \leq \nu \leq M_{1}, 1 \leq \mu \leq M_{2} .
$$

Le lemme 1 dit que c'est impossible si on impose $M_{1}>D_{1}$ et $M_{2}>$ $D_{0}\left(1+D_{1}\right)$ c'est à dire $S>T_{1}-1$ et $S^{2}>\left(T_{0}-1\right) \cdot T_{1}$. 
On va donc imposer les deux contraintes suivantes

$$
\begin{array}{ll}
(\mathcal{C} 1) & S \geq T_{1}, \\
(\mathcal{C} 2) & S^{2} \geq T_{0} T_{1},
\end{array}
$$

et sous ces contraintes on sait maintenant que la matrice $\mathcal{M}$ est de rang maximal c'est à dire de rang égal à $\min \left(T_{0} T_{1} ; 4 S^{2}\right)$ et donc de rang égal à $T_{0} T_{1}$ (compte tenu de $\mathcal{C} 2$ ).

On peut donc extraire de $\mathcal{S}$ une partie $\mathcal{S}_{0}$ ayant $T_{0} T_{1}$ éléments telle que le déterminant $\Delta=\operatorname{det}\left(\phi_{t}\left(z_{s}\right)\right)_{t \in \mathcal{T}, s \in \mathcal{S}_{0}}$ soit non nul. On va maintenant majorer et minorer $|\Delta|$.

- Second point - Majoration de $|\Delta|$.

Je pose $L:=T_{0} T_{1}$ et $\psi(z):=\operatorname{det}\left(\phi_{t}\left(z . z_{s}\right)\right)_{t \in \mathcal{T}, s \in \mathcal{S}_{0}}$. Le lemme 3 dit que cette fonction $\psi$ a un zéro à l'origine de multiplicité supérieure ou égale à $L(L-1) / 2$.

On va vérifier que $\psi$ est un polynôme exponentiel et le lemme 2 permettra alors de majorer $|\psi(1)|$ c'est à dire $|\Delta|$.

On part de $\psi(z)=\operatorname{det}\left(\phi_{t}\left(z . z_{s}\right)\right)_{t \in \mathcal{T}, s \in \mathcal{S}_{0}}=\operatorname{det}\left(\left(z . z_{s}\right)^{t_{0}} \cdot e^{t_{1} v z z_{s}}\right)_{t \in \mathcal{T}, s \in \mathcal{S}_{0}}$ que l'on développe :

$$
\psi(z)=\sum_{\sigma} \varepsilon(\sigma) \prod_{t_{0}<T_{0}} \prod_{t_{1}<T_{1}}\left(z . z_{\sigma(t)}\right)^{t_{0}} e^{t_{1} v z z_{\sigma(t)}}
$$

où $\sigma$ parcourt l'ensemble des bijections de $\mathcal{T}$ dans $\mathcal{S}_{0}$ et $\varepsilon(\sigma)$ est la signature de $\sigma$ prise en fonction des ordres choisis sur $\mathcal{T}$ et $\mathcal{S}$. Comme $\Pi_{t_{0}} \Pi_{t_{1}} z^{t_{0}}=$ $z^{\frac{1}{2} T_{0}\left(T_{0}-1\right) T_{1}}$ il vient :

$$
\psi(z)=\sum_{\sigma} \varepsilon(\sigma) z^{\frac{1}{2} T_{0}\left(T_{0}-1\right) T_{1}}\left(\prod_{t_{0}<T_{0}} \prod_{t_{1}<T_{1}} z_{\sigma(t)}^{t_{0}}\right) \cdot e^{\sum_{t_{0}<T_{0}} \sum_{t_{1}<T_{1}} t_{1} v z z_{\sigma(t)}} .
$$

En posant $a(\sigma):=\prod_{t_{0}<T_{0}} \prod_{t_{1}<T_{1}} z_{\sigma(t)}^{t_{0}}, \quad b(\sigma):=\sum_{t_{0}<T_{0}} \sum_{t_{1}<T_{1}} t_{1} v z_{\sigma(t)}$ on a ainsi :

$$
\psi(z)=\sum_{\sigma} \varepsilon(\sigma) \cdot a(\sigma) \cdot z^{\frac{1}{2} T_{0}\left(T_{0}-1\right) T_{1}} \cdot e^{b(\sigma) z} .
$$

On a donc bien un polynôme exponentiel, de la forme

$$
\sum_{j=1}^{t} a_{j} . z^{s} \cdot e^{\omega_{j} z} \text { avec } s=\frac{1}{2} T_{0}\left(T_{0}-1\right) T_{1}, t=L ! .
$$

On applique le lemme 2 avec $z_{0}=1$; pour $\rho \geq 1$ on obtient alors :

$$
|\psi(1)| \leq \rho^{-L(L-1) / 2} \cdot \sum_{\sigma}|a(\sigma)| \cdot \rho^{\frac{1}{2} T_{0}\left(T_{0}-1\right) T_{1}} \cdot e^{|b(\sigma)| \rho} .
$$


Pour $s \in \mathcal{S}$ on a $\left|z_{s}\right|=\left|s_{1} \alpha+s_{2} \bar{\alpha}\right| \leq\left(s_{1}+s_{2}\right)|\alpha| \leq 4 S|\alpha|$. Je noterai $r:=4 S|\alpha|$ (et donc tous les points $z_{s}, s \in \mathcal{S}$, sont dans le disque $|z| \leq r$ ). J'introduis un paramètre réel $R \geq r$ et je prends $\rho:=\frac{R}{r}$. On a donc une nouvelle contrainte :

$$
\text { (C3) } \quad R \geq 4|\alpha| S .
$$

On a alors pour tout $\sigma$ :

$$
\text { * }|a(\sigma)|=\prod_{t_{0}<T_{0}} \prod_{t_{1}<T_{1}}\left|z_{\sigma(t)}^{t_{0}}\right| \leq \prod_{t_{0}<T_{0}} \prod_{t_{1}<T_{1}} r^{t_{0}}=r^{\frac{1}{2} T_{0}\left(T_{0}-1\right) T_{1}} ;
$$

donc :

$$
|a(\sigma)| \cdot \rho^{\frac{1}{2} T_{0}\left(T_{0}-1\right) T_{1}} \leq(r \rho)^{\frac{1}{2} T_{0}\left(T_{0}-1\right) T_{1}}=R^{\frac{1}{2} L\left(T_{0}-1\right)} ;
$$

et donc (quitte à supposer $S$ assez grand pour avoir $r \geq 1$ ) :

$$
\begin{aligned}
*|b(\sigma)| & \leq \sum_{t_{0}<T_{0}} \sum_{t_{1}<T_{1}} t_{1} \cdot|v| \cdot\left|z_{\sigma(t)}\right| \\
& \leq|v| \cdot r \cdot\left(\sum_{t_{0}<T_{0}} \sum_{t_{1}<T_{1}} t_{1}\right) \text { et donc : } \\
& \leq|v| \cdot r \cdot \frac{1}{2} T_{0} T_{1}\left(T_{1}-1\right) ; \\
& |b(\sigma)| \rho \leq|v| \cdot R \cdot \frac{1}{2} T_{0} T_{1}\left(T_{1}-1\right) \leq|v| R L T_{1} .
\end{aligned}
$$

Au total on a donc en revenant à (5) :

$$
|\triangle|=|\psi(1)| \leq\left(\frac{R}{r}\right)^{-L(L-1) / 2} \cdot(L !) \cdot R^{L T_{0}} \cdot e^{|v| R L T_{1}} .
$$

\section{- Troisième point - Minoration de $|\triangle|$.}

Jusqu'ici l'hypothèse que $e^{v \alpha}$ et $\alpha$ sont algébriques n'a pas été utilisée. Par contre elle va l'être maintenant. Elle permet de dire que $\Delta$ est un nombre algébrique (non nul par construction) qui s'écrit comme une expression $\mathbb{Z}$-polynômiale en $\left(\alpha, \bar{\alpha}, e^{v \alpha}, e^{v \bar{\alpha}}\right)$ et que l'on peut donc minorer en module à l'aide du lemme 4.

Rappelons l'expression de $\triangle$ :

$$
\triangle=\operatorname{det}\left(\left(s_{1} \alpha+s_{2} \bar{\alpha}\right)^{t_{0}} \cdot\left(e^{v \alpha}\right)^{t_{1} s_{1}} \cdot\left(e^{v \bar{\alpha}}\right)^{t_{1} s_{2}}\right)_{t \in \mathcal{T}, s \in \mathcal{S}_{0}} .
$$

Pour $(t, s) \in \mathcal{T} \times \mathcal{S}$ j'introduis $Q_{t s} \in \mathbb{Z}\left[Y_{1}, Y_{2}, Y_{3}, Y_{4}\right]$ défini par :

$$
Q_{t s}\left(Y_{1}, Y_{2}, Y_{3}, Y_{4}\right):=\left(s_{1} Y_{1}+s_{2} Y_{2}\right)^{t_{0}} \cdot Y_{3}^{t_{1} s_{1}} \cdot Y_{4}^{t_{1} s_{2}} .
$$

Et $Q \in \mathbb{Z}\left[Y_{1}, Y_{2}, Y_{3}, Y_{4}\right]$ est défini par :

$$
Q\left(Y_{1}, Y_{2}, Y_{3}, Y_{4}\right):=\operatorname{det}\left(Q_{t s}\right)_{t \in \mathcal{T}, s \in \mathcal{S}_{0}}=\sum_{\sigma} \varepsilon(\sigma) . \prod_{t_{0}<T_{0}} \prod_{t_{1}<T_{1}} Q_{t \sigma(t)} .
$$


Avec ces notations on a alors

$$
\triangle=\operatorname{det}\left(Q_{t s}\left(\alpha, \bar{\alpha}, e^{v \alpha}, e^{v \bar{\alpha}}\right)\right)_{t \in \mathcal{T}, s \in \mathcal{S}_{0}}=Q\left(\alpha, \bar{\alpha}, e^{v \alpha}, e^{v \bar{\alpha}}\right) .
$$

En notant $d$ le degré du corps de nombres $\mathbb{Q}\left(\alpha, \bar{\alpha}, e^{v \alpha}, e^{v \bar{\alpha}}\right)$, le lemme 4 donne alors :

$$
\begin{aligned}
\log |\triangle| \geq- & d \log L(Q)-d\left(h(\alpha) \operatorname{deg}_{Y_{1}}(Q)\right. \\
& \left.+h(\bar{\alpha}) \operatorname{deg}_{Y_{2}}(Q)+h\left(e^{v \alpha}\right) \operatorname{deg}_{Y_{3}}(Q)+h\left(e^{v \bar{\alpha}}\right) \operatorname{deg}_{Y_{4}}(Q)\right) .
\end{aligned}
$$

* Estimation de $L(Q)$. On sait (voir [Wa 2] p.29) que la longueur $L($.) vérifie $L(f+g) \leq L(f)+L(g), L(f . g) \leq L(f) . L(g)$. En revenant à la définition $\mathrm{du}$ polynôme $Q$ on a donc :

$$
L(Q) \leq \sum_{\sigma}\left(\prod_{t_{0}<T_{0}} \prod_{t_{1}<T_{1}} L\left(Q_{t \sigma(t)}\right)\right) \leq(L !)\left(\max _{t \in \mathcal{T}, s \in \mathcal{S}} L\left(Q_{t s}\right)\right)^{T_{0} T_{1}} .
$$

Et par ailleurs pour $t \in \mathcal{T}$ et $s \in \mathcal{S}$ on a

$$
\begin{aligned}
L\left(Q_{t s}\right) & \leq\left(L\left(s_{1} Y_{1}+s_{2} Y_{2}\right)\right)^{t_{0}} \\
& \leq\left(\left|s_{1}\right|+\left|s_{2}\right|\right)^{t_{0}} \\
& \leq(4 S)^{T_{0}} .
\end{aligned}
$$

Donc au total : $L(Q) \leq(L !)(4 S)^{T_{0} L}$.

* Estimation des degrés partiels de $Q$. Pour $t \in \mathcal{T}, s \in \mathcal{S}$ on a :

$$
\begin{array}{lll}
\operatorname{deg}_{Y_{1}}\left(Q_{t s}\right)<T_{0}, & \operatorname{deg}_{Y_{2}}\left(Q_{t s}\right)<T_{0}, \\
\operatorname{deg}_{Y_{3}}\left(Q_{t s}\right)<2 S T_{1}, & \operatorname{deg}_{Y_{4}}\left(Q_{t s}\right)<2 S T_{1} .
\end{array}
$$

A partir $\operatorname{de} \operatorname{deg}_{Y_{i}}(Q) \leq \max _{s \in S} \sum_{t_{0}<T_{0}} \sum_{t_{1}<T_{1}} \operatorname{deg}_{Y_{i}}\left(Q_{t s}\right), i \in\{1,2,3,4\}$, on obtient :

$$
\begin{array}{ll}
\operatorname{deg}_{Y_{1}}(Q) \leq L T_{0}, & \operatorname{deg}_{Y_{2}}(Q) \leq L T_{0}, \\
\operatorname{deg}_{Y_{3}}(Q) \leq 2 S T_{1} L, & \operatorname{deg}_{Y_{4}}(Q) \leq 2 S T_{1} L .
\end{array}
$$

Ces estimations élémentaires sont des cas particuliers du lemme 3.15 de [Wa 3], page 85.

* Donc au total (7) s'écrit, avec ces estimations :

$$
\log |\triangle| \geq-d\left(\log (L !)+T_{0} L \log (4 S)+2 h(\alpha) \cdot L T_{0}+4 h\left(e^{v \alpha}\right) \cdot S T_{1} L\right) .
$$

On a donc en majorant $\log (L !)$ par $L \log L\left(\right.$ rappel $\left.: L=T_{0} T_{1}\right)$ :

$$
\log |\triangle| \geq-c_{1} L\left(T_{0} \log (4 S)+S T_{1}\right)
$$

où $c_{1}>0$ ne dépend pas des paramètres $T_{0}, T_{1}, S, R$ (il en sera de même pour les "constantes" $c_{2}, c_{3}$ qui apparaitront ensuite). 


\section{- Quatrième point - Contradiction finale.}

On met bout à bout la majoration (6) et la minoration (8) de $|\triangle|$. On a donc :

$$
\begin{aligned}
& -c_{1} L\left(T_{0} \log (4 S)+S T_{1}\right) \leq-\frac{L(L-1)}{2} \log \left(\frac{R}{r}\right) \\
& \quad+\log (L !)+L T_{0} \log R+|v| R L T_{1} .
\end{aligned}
$$

On majore $\log (L !)$ par $L \log L$ et on simplifie par $L ;$ il reste :

$$
\frac{L-1}{2} \cdot \log \left(\frac{R}{r}\right) \leq c_{1}\left(T_{0} \log (4 S)+S T_{1}\right)+\log L+T_{0} \log R+|v| R T_{1}
$$

et donc a fortiori, pour une constante $c_{2}$ ad - hoc :

$$
L \log \left(\frac{R}{r}\right) \leq c_{2}\left(T_{0} \log (4 S)+S T_{1}+T_{0} \log R+R T_{1}\right) .
$$

Comme on a $R \geq r=4|\alpha| S$ on en déduit, pour une constante $c_{3}$ ad - hoc :

$$
L \log \left(\frac{R}{r}\right) \leq c_{3}\left(T_{0} \log R+T_{1} R\right) .
$$

Pour obtenir une contradiction il suffit donc d'imposer la contrainte finale :

$$
T_{0} T_{1} \log \left(\frac{R}{r}\right)>c_{3} \cdot\left(T_{0} \log R+T_{1} R\right) .
$$

Au total on aura donc obtenu la contradiction cherchée si on peut choisir les paramètres $\left(T_{0}, T_{1}, S, R\right)$ de façon à satisfaire les quatre contraintes :

$$
\begin{cases}(\mathcal{C} 1) & T_{1} \leq S \\ (\mathcal{C} 2) & T_{0} T_{1} \leq S^{2} \\ (\mathcal{C} 3) & 4|\alpha| S \leq R \\ (\mathcal{C} 4) & c_{3}\left(T_{0} \log R+T_{1} R\right)<T_{0} T_{1} \log \left(\frac{R}{4|\alpha| S}\right) .\end{cases}
$$

Par exemple à $S$ entier on associe $T_{0}:=[S \log S], T_{1}:=\left[(\log S)^{2}\right]$, où [.] note la partie entière, $R:=e r=e(4|\alpha| S)$. Pour tout $S$ assez grand, ces paramètres satisfont les quatre contraintes ci-dessus, ce qui termine la démonstration.

\subsection{Quelles sont les propriétés qui ont été utilisées?}

Les lemmes 1 et 4 sont purement algébriques et arithmétiques, et totalement indépendants de la fonction exponentielle. Le lemme 3 ne fait intervenir que le développement à l'origine des fonctions entières et la multilinéarité du déterminant. 
Le lemme 2, le seul qui fasse intervenir $e^{z}$, n'utilise que le développement $e^{z}=\sum_{n \geq 0} \frac{z^{n}}{n !}$. Et la preuve elle-même utilise, en plus, la propriété $\exp (a+b)=$ $\exp (a) \cdot \exp (b)$

Finalement le recours à l'analyse apparait très limité : les développements en série entière et quelques estimations élémentaires! Aucun théorème profond d'analyse complexe (ou d'analyse réelle) n'intervient.

\section{Deux cas particuliers de la conjecture de Schanuel}

Il existe plusieurs conjectures classiques portant sur la transcendance ou l'indépendance algébrique de valeurs de la fonction exponentielle, et il n'y a pas eu de progrès notable depuis longtemps (curieusement le seul succès récent, l'indépendance algébrique de $\pi$ et $e^{\pi}$ obtenue par Y.V. Nesterenko en 1996, ne fait pas du tout intervenir la fonction exponentielle dans sa preuve!).

La tentation est donc forte de s'attaquer à des cas particuliers de ces conjectures plutôt qu'aux conjectures générales. Par exemple dans [Di], à la suite de travaux de D. Bertrand, j'ai proposé un certain nombre de conjectures qui peuvent être lues avec un point de vue "modulaire" ou avec un point de vue "exponentielle" ; l'espoir est bien sûr qu'en multipliant les angles d'attaque on finisse par trouver le bon, mais pour l'instant ce n'est pas le cas. Ici je vais proposer deux cas particuliers de la conjecture de Schanuel, que je trouve particulièrement intéressants.

\subsection{Une première conjecture.}

C'est la conjecture évoquée au paragraphe 1 . On va voir qu'elle se déduit de la conjecture de Schanuel ([Wa 3], p. 16), et même plus précisément de la conjecture d'indépendance algébrique des logarithmes de nombres algébriques ([Wa 3], p. 16) ; c'est aussi un cas particulier de la conjecture forte des quatre exponentielles déjà évoquée au paragraphe 2 ([Wa 3], p. $399)$.

Conjecture de Schanuel. Soient $u_{1}, \ldots, u_{n}$ des nombres complexes $\mathbb{Q}$ linéairement indépendants. On a alors :

$$
\operatorname{degtr}_{\mathbb{Q}} \mathbb{Q}\left(u_{1}, \ldots, u_{n}, e^{u_{1}}, \ldots, e^{u_{n}}\right) \geq n .
$$

Conjecture $\mathcal{C}(|\mathbf{u}|)$. Soit $u \in \mathbb{C} \backslash\{0\}$. Si $|u|$ est algébrique, alors $e^{u}$ est transcendant.

Proposition 1. $\mathcal{C}(|u|)$ est une conséquence de la conjecture de Schanuel.

Preuve de la proposition 1.

Je suppose $|u|$ algébrique non nul et je distingue deux cas. 
Cas $1: u \in \mathbb{R} \cup i \mathbb{R}$. On a alors $|u|= \pm u$ (si $u \in \mathbb{R}$ ) ou $|u|= \pm i u$ (si $u \in i \mathbb{R}$ ); en conséquence $u$ lui-même est algébrique, et le théorème de Hermite-Lindemann dit que $e^{u}$ est transcendant.

Cas 2: $u \notin \mathbb{R} \cup i \mathbb{R}$. Dans ce cas $(u, \bar{u})$ est $\mathbb{Q}$-libre et par la conjecture de Schanuel on sait que $\operatorname{degtr}_{\mathbb{Q}} \mathbb{Q}\left(u, \bar{u}, e^{u}, e^{\bar{u}}\right) \geq 2$. Si $e^{u}$ était algébrique, $e^{\bar{u}}=\overline{e^{u}}$ le serait aussi et il resterait $\operatorname{degtr}_{\mathbb{Q}} \mathbb{Q}(u, \bar{u}) \geq 2$, ce qui est faux puisque par hypothèse $u . \bar{u} \in \overline{\mathbb{Q}}$. C'est donc que $e^{u}$ est transcendant.

Le fait que $\mathcal{C}(|u|)$ soit un cas particulier de la conjecture de Schanuel lui donne la crédibilité nécessaire; mais on peut se dire que le point de vue "Schanuel" n'est peut être pas le bon, ne serait-ce que parce que l'on passe par un résultat d'indépendance algébrique pour établir un "simple" résultat de transcendance. Du coup cela pose la question, qui me semble intéressante, de la méthode : comment aborder $\mathcal{C}(|u|)$ ? Comment utiliser l'hypothèse $|u| \in \overline{\mathbb{Q}}$ ? Comment faire apparaître $|u|$ ? Peut-on envisager d'utiliser des fonctions comme $z \mapsto \bar{z}, z \mapsto|z| \ldots$ ? C'est ce type de question qui donne à mon goût de l'intérêt à $\mathcal{C}(|u|)$.

$\mathrm{Au}$ demeurant on peut énoncer d'autres conjectures "raisonnables" faisant apparaître le module ou la conjugaison complexe (voir par exemple [Wa $3]$ pages 15,399 et 614 , exercice 15.16 ) ; et pour elles aussi les questions précédentes sont posées.

\subsection{Une seconde conjecture.}

J'ai mis en relief la conjecture $\mathcal{C}(|u|)$ pour son caractère esthétique et parce qu'elle conduit à des questions de méthode qui me semblent intéressantes. Mais on peut évidemment m'objecter que la formulation choisie n'est pas bonne, précisément parce qu'elle pousse dans une impasse! Par exemple la conjecture suivante, qui est plus forte que $\mathcal{C}(|u|)$, ne fait pas du tout apparaitre de module et est certainement tout aussi intéressante; elle se déduit elle aussi de la conjecture de Schanuel, et de la conjecture forte des quatre exponentielles.

Conjecture $\mathcal{C}\left(\mathbf{u}_{1}, \mathbf{u}_{2}\right)$. Soient $u_{1}$ et $u_{2}$ deux nombres complexes non nuls. On a alors pour tout $\beta \in \overline{\mathbb{Q}} \backslash\{0\}$ :

$$
\begin{aligned}
& \text { a) } \operatorname{degtr}_{\mathbb{Q}} \mathbb{Q}\left(u_{1} u_{2}, e^{u_{1}}, e^{u_{2}}\right) \geq 1, \\
& \text { b) } \operatorname{degtr}_{\mathbb{Q}} \mathbb{Q}\left(e^{\beta u_{1} u_{2}}, e^{u_{1}}, e^{u_{2}}\right) \geq 1 .
\end{aligned}
$$

Les assertions (a) et (b) sont bien sûr vraies lorsque $u_{1}$ ou $u_{2}$ est algébrique.

Proposition 2. $\mathcal{C}\left(u_{1}, u_{2}\right)$ est une conséquence de la conjecture de Schanuel.

Preuve de la proposition 2.

Contrairement à la proposition 1, ici la conjugaison complexe n'intervient pas. 
Assertion (a). On suppose $e^{u_{1}}$ et $e^{u_{2}}$ algébriques et on cherche à démontrer que $u_{1} u_{2}$ est transcendant. On distingue deux cas.

Cas $1:\left(\mathbf{u}_{1}, \mathbf{u}_{2}\right)$ est $\mathbb{Q}$-libre. La conjecture de Schanuel appliquée à $\left(u_{1}, u_{2}\right)$ dit que $u_{1}$ et $u_{2}$ sont algébriquement indépendants ; en conséquence $u_{1} u_{2}$ est transcendant.

Cas $2:\left(\mathbf{u}_{1}, \mathbf{u}_{2}\right)$ est $\mathbb{Q}$-liée. On peut se ramener au cas $u_{1}=u_{2}$. Puisque $e^{u_{1}}$ est algébrique, on sait que $u_{1}$ est transcendant ; et donc à fortiori $u_{1} u_{2}=$ $u_{1}^{2}$ l'est aussi.

Assertion (b). On suppose $e^{u_{1}}, e^{u_{2}}, e^{\beta u_{1} u_{2}}$ algébriques et on cherche une contradiction. Si $\left(u_{1}, u_{2}, \beta u_{1} u_{2}\right)$ était $\mathbb{Q}$-libre, la conjecture de Schanuel donnerait $\operatorname{deg}_{\mathbb{Q}} \mathbb{Q}\left(u_{1}, u_{2}, \beta u_{1} u_{2}\right)=3$, ce qui est faux. Donc $\left(u_{1}, u_{2}, \beta u_{1} u_{2}\right)$ est $\mathbb{Q}$-liée : il existe $a, b, c$ dans $\mathbb{Q}$, non tous nuls, vérifiant $a u_{1}+b u_{2}=$ $c \beta u_{1} u_{2}$.

Cas $1: \mathbf{c}=\mathbf{0}$. Il reste $a u_{1}+b u_{2}=0,(a, b) \neq(0,0)$. On a donc $a \neq$ $0, b \neq 0$ et on peut se ramener au cas $u_{1}=u_{2}$. On applique la conjecture de Schanuel à $\left(u_{1}, \beta u_{1}^{2}\right)$ qui est $\mathbb{Q}$-libre car $u_{1}$ est transcendant; on obtient $\operatorname{degtr}_{\mathbb{Q}} \mathbb{Q}\left(u_{1}, \beta u_{1}^{2}\right)=2$, ce qui est faux.

Cas $2: \mathbf{c} \neq \mathbf{0}$.

sous-cas $1:\left(\mathbf{u}_{1}, \mathbf{u}_{2}\right)$ est $\mathbb{Q}$-libre. On applique la conjecture de Schanuel à $\left(u_{1}, u_{2}\right)$; elle dit que $u_{1}$ et $u_{2}$ sont algébriquement indépendants, ce qui est incompatible avec la relation $a u_{1}+b u_{2}=c \beta u_{1} u_{2}$.

sous-cas $2:\left(\mathbf{u}_{1}, \mathbf{u}_{2}\right)$ est $\mathbb{Q}$-liée. Dans ce cas, il existe $d \in \mathbb{Q}$ tel que $u_{2}=d u_{1}$; et la relation $c \beta u_{1} u_{2}=a u_{1}+b u_{2}$ donne $c \beta u_{2}=a+b d$. Mais ceci est impossible puisque $u_{2}$ est transcendant

Je ne sais pas démontrer la conjecture $\mathcal{C}\left(u_{1}, u_{2}\right)$ en toute généralité, mais je vais montrer grâce au théorème 3 (la conjugaison complexe réapparait) qu'elle est vraie dans un certain nombre de cas. Les notations $\mathcal{L}$ et $\widetilde{\mathcal{L}}$ ont été introduites au paragraphe 2.

Théorème 6. Soient $u_{1}, u_{2}$ deux nombres complexes non nuls. On suppose $u_{1} \in \mathbb{R} \cup i \mathbb{R}, u_{2} \notin \mathbb{R} \cup i \mathbb{R}$. Alors on a pour tout $\beta \in \overline{\mathbb{Q}} \backslash\{0\}$ :

$$
\begin{aligned}
& \text { a) } \operatorname{deg} \operatorname{tr}_{\mathbb{Q}} \mathbb{Q}\left(u_{1} u_{2}, e^{u_{1}}, e^{u_{2}}\right) \geq 1, \\
& \text { b) } \operatorname{deg} \operatorname{tr}_{\mathbb{Q}} \mathbb{Q}\left(e^{\beta u_{1} u_{2}}, e^{u_{1}}, e^{u_{2}}\right) \geq 1 .
\end{aligned}
$$

Plus précisément, si $e^{u_{1}}$ et $e^{u_{2}}$ sont algébriques alors $u_{1} u_{2}$ 'est pas dans $\widetilde{\mathcal{L}}$

Malheureusement les hypothèses interdisent de prendre $u_{1}=u, u_{2}=\bar{u}$ et donc le théorème 6 ne dit rien $\operatorname{de} \mathcal{C}(|u|)$. On peut illustrer le (a) en disant par exemple que $\exp \left(\frac{1+i}{\pi}\right)$ est transcendant (prendre $u_{1}=2 i \pi, u_{2}=\frac{1+i}{\pi}$ ).

Preuve du théorème 6 . 
Le théorème 6 est une simple conséquence du théorème 3 , point 2 . On suppose $e^{u_{1}}$ et $e^{u_{2}}$ algébriques (sinon le résultat est acquis); donc $u_{1}$ et $u_{2}$ sont des éléments de $\mathcal{L} \backslash\{0\}$. Le théorème 3-(2) appliqué à $\lambda_{0}=u_{1}, \lambda_{1}=u_{2}$ dit que $u_{1} u_{2}$ n'est pas dans $\widetilde{\mathcal{L}}$. A fortiori $u_{1} u_{2}$ n'est pas dans $\overline{\mathbb{Q}}$ et cela prouve (a); de même $\beta u_{1} u_{2}$ n'est pas dans $\mathcal{L}$, donc $\exp \left(\beta u_{1} u_{2}\right)$ est transcendant, et cela prouve (b).

Les formulations (a) et (b) illustrent $u_{1} u_{2} \notin \widetilde{\mathcal{L}}$ mais ne résument pas l'information, loin de là.

Damien Roy a donné une formulation de la conjecture de Schanuel de nature purement algébrique et assez proche des critères d'indépendance algébriques avec multiplicités (voir [Ro] ou [Wa 3], page 605). Peut-on, dans le même esprit, reformuler de façon purement algébrique les conjectures $\mathcal{C}(|u|)$ et $\mathcal{C}\left(u_{1}, u_{2}\right)$ ?

J'ai déjà indiqué, et le lecteur peut le vérifier sans peine, que les conjectures $\mathcal{C}(|u|)$ et $\mathcal{C}\left(u_{1}, u_{2}\right)$ sont des conséquences de la conjecture forte des quatre exponentielles ([Wa 3], p. 399). On est donc dans des situations que l'on sait attaquer avec les outils actuels de la transcendance; malheureusement on ne sait pas conclure en toute généralité. Tous les problèmes de transcendance de type "quatre exponentielles" résistent et ils constituent donc un terrain de réflexion qui mérite attention.

\section{Bibliographie}

[Di] G. DiAz, La conjecture des quatre exponentielles et les conjectures de D. Bertrand sur la fonction modulaire. J. Théorie des Nombres de Bordeaux 9 (1997), 229-245.

[Ge - Li] A.0. GeL'Fond, Ju.V. LINNIK, Méthodes élémentaires dans la théorie analytique des Nombres. Monographies internationales de Mathématiques modernes vol. 6, GauthierVillars, Paris, 1965.

[La] M. LAURENT, Sur quelques résultats récents de transcendance. Astérisque $\mathbf{n}^{\circ} \mathbf{1 9 8 - 2 0 0}^{19}$ (1991), 209-230.

[Ro] D. RoY, An arithmetic criterion for the values of the exponential function. Acta Arith. XCVII. 2 (2001), 183-194.

[Wa 1] M. WALDSCHMIDT, Une méthode élémentaire dans la théorie des nombres transcendants I, II. Séminaire DPP, $\mathbf{n}^{\circ} \mathbf{G 1}$ et G5 (1972/73).

[Wa 2] M. WALDSChMidT, Nombres transcendants. Lecture Notes in Mathematics vol. 402, Springer-Verlag, Berlin-New York, 1974.

[Wa 3] M. WALDSCHMIDT, Diophantine approximation on linear algebraic groups. Grundlerhen der mathematischen Wissenschaften, vol. 326, Springer-Verlag, Berlin-Heidelbeg-New York, 2000.

Guy Diaz

Université de Saint-Etienne

LARAL

23 rue du Dr Paul Michelon

42023 Saint-Etienne Cedex 2, FRANCE 